\title{
First operation of a harmonic lasing self-seeded free electron laser
}

\author{
E. A. Schneidmiller, B. Faatz, M. Kuhlmann, J. Rönsch-Schulenburg, S. Schreiber, \\ M. Tischer, and M. V. Yurkov \\ Deutsches Elektronen-Synchrotron (DESY), Notkestrasse 85, D-22607 Hamburg, Germany
}

(Received 12 December 2016; published 15 February 2017)

\begin{abstract}
Harmonic lasing is a possible mode of operation of X-ray FEL user facilities that allows us to provide brilliant beams of higher energy photons for user experiments. Another useful application of harmonic lasing is the so-called harmonic lasing self-seeded free electron laser (HLSS FEL) that allows the improvement of spectral brightness of these facilities. In the past, harmonic lasing has been demonstrated in the FEL oscillators in infrared and visible wavelength ranges, but not in high-gain FELs and not at short wavelengths. In this paper we report on the first evidence of the harmonic lasing and the first operation of the HLSS FEL at the soft X-ray FEL user facility FLASH in the wavelength range between $4.5 \mathrm{~nm}$ and $15 \mathrm{~nm}$. Spectral brightness was improved in comparison with self-amplified spontaneous emission (SASE) FEL by a factor of six in the exponential gain regime. A better performance of HLSS FEL with respect to SASE FEL in the post-saturation regime with a tapered undulator was observed as well. The first demonstration of harmonic lasing in a high-gain FEL and at short wavelengths paves the way for a variety of applications of this new operation mode in X-ray FELs.
\end{abstract}

DOI: $10.1103 /$ PhysRevAccelBeams.20.020705

\section{INTRODUCTION}

Successful operation of X-ray free electron lasers (FELs) [1-3], based on self-amplified spontaneous emission (SASE) principle [4], down to an Ångström regime opens up new horizons for photon science. Even shorter wavelengths are requested by the scientific community.

One of the most promising ways to extend the photon energy range of high-gain X-ray FELs is to use harmonic lasing which is the FEL instability at an odd harmonic of the planar undulator [5-9] developing independently from the lasing at the fundamental. Contrary to the nonlinear harmonic generation [1,6,7,10-13] (which is driven by the fundamental in the vicinity of saturation), harmonic lasing can provide much more intense, stable, and narrow-band radiation if the fundamental is suppressed. The most attractive feature of saturated harmonic lasing is that the spectral brightness of a harmonic is comparable to that of the fundamental [9].

Another interesting option, proposed in [9], is the possibility to improve spectral brightness of an X-ray FEL by the combined lasing on a harmonic in the first part of the undulator (with an increased undulator parameter $K$ ) and on the fundamental in the second part of the undulator. Later this concept was named harmonic lasing

\footnotetext{
*evgeny.schneidmiller@desy.de
}

Published by the American Physical Society under the terms of the Creative Commons Attribution 4.0 International license. Further distribution of this work must maintain attribution to the author(s) and the published article's title, journal citation, and DOI. self-seeded FEL (HLSS FEL) [14]. Even though this scheme is not expected to provide an ultimate monochromatization of the FEL radiation as do self-seeding schemes using optical elements [15-17], it has other advantages that we briefly discuss below in the paper.

Harmonic lasing was initially proposed for FEL oscillators [18] and was tested experimentally in infrared and visible wavelength ranges [19-22]. It was, however, never demonstrated in high-gain FELs and at a short wavelength. In this paper we present the first successful demonstration of this effect at the second branch of the soft X-ray FEL user facility FLASH [23] where we managed to run HLSS FEL in the wavelength range between $4.5 \mathrm{~nm}$ and $15 \mathrm{~nm}$.

\section{HARMONIC LASING}

Harmonic lasing in single-pass high-gain FELs [5-9] is the amplification process of higher odd harmonics developing independently of each other (and of the fundamental harmonic) in the exponential gain regime. In the case of a SASE FEL the fluctuations of the beam current with frequency components in the vicinity of a wavelength

$$
\lambda_{h}=\frac{\lambda_{\mathrm{w}}\left(1+K^{2}\right)}{2 h \gamma^{2}} \quad h=1,3,5 \ldots
$$

serve as an input signal for amplification process. Here $\lambda_{\mathrm{w}}$ is the undulator period, $\gamma$ is relativistic factor, $h$ is harmonic number, and $K$ is the rms undulator parameter:

$$
K=0.934 \lambda_{\mathrm{w}}[\mathrm{cm}] B_{\mathrm{rms}}[\mathrm{T}]
$$


$B_{\mathrm{rms}}$ being the rms undulator field (peak field divided by $\sqrt{2}$ for a planar undulator with the sinusoidal field).

An advantage of harmonic lasing over lasing on the fundamental at the same wavelength can be demonstrated for the case of a gap-tunable undulator. In this case one uses a higher $K$-value for harmonic lasing, i.e. for the lasing on the fundamental one has to reduce $K$ to the value $K_{\text {re }}$ :

$$
K_{\mathrm{re}}^{2}=\frac{1+K^{2}}{h}-1
$$

Obviously, $K$ must be larger than $\sqrt{h-1}$.

Then one can derive a ratio of the gain length of the fundamental, $L_{g}^{(1)}$, to the gain length of a harmonic $L_{g}^{(h)}[9]$ :

$$
\frac{L_{g}^{(1)}}{L_{g}^{(h)}}=\frac{h^{1 / 2} K A_{J J h}(K)}{K_{\mathrm{re}} A_{J J 1}\left(K_{\mathrm{re}}\right)} .
$$

Here $A_{J J h}(K)=J_{(h-1) / 2}\left(\frac{h K^{2}}{2\left(1+K^{2}\right)}\right)-J_{(h+1) / 2}\left(\frac{h K^{2}}{2\left(1+K^{2}\right)}\right)$ is the coupling factor for harmonics with $J_{n}$ being Bessel functions. The coupling factors for the $1 \mathrm{st}, 3 \mathrm{rd}$, and 5 th harmonics are shown in Fig. 1.

The formula (3) is obtained in the frame of the threedimensional theory including diffraction of the radiation, emittance, betatron motion (and for an optimized betafunction) but assuming a negligible energy spread. The plot of the ratio of gain lengths (3) is presented in Fig. 2. It is clearly seen that harmonic lasing has always a shorter gain length under above mentioned conditions (and the ratio is larger than that obtained in the one-dimensional model [8]). The ratio shown in Fig. 2 starts to diverge rapidly for the values of $K$ approaching $\sqrt{2}$, and lasing at the fundamental becomes impossible below this point. However, there still

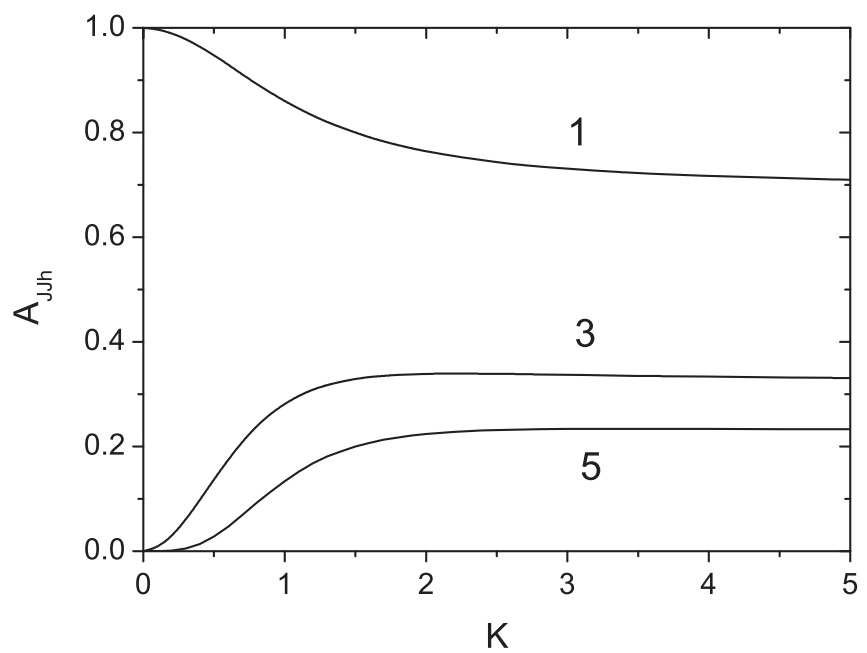

FIG. 1. Coupling factors for the 1st, 3rd, and 5th harmonics (denoted with 1, 3, and 5, correspondingly) versus rms undulator parameter.

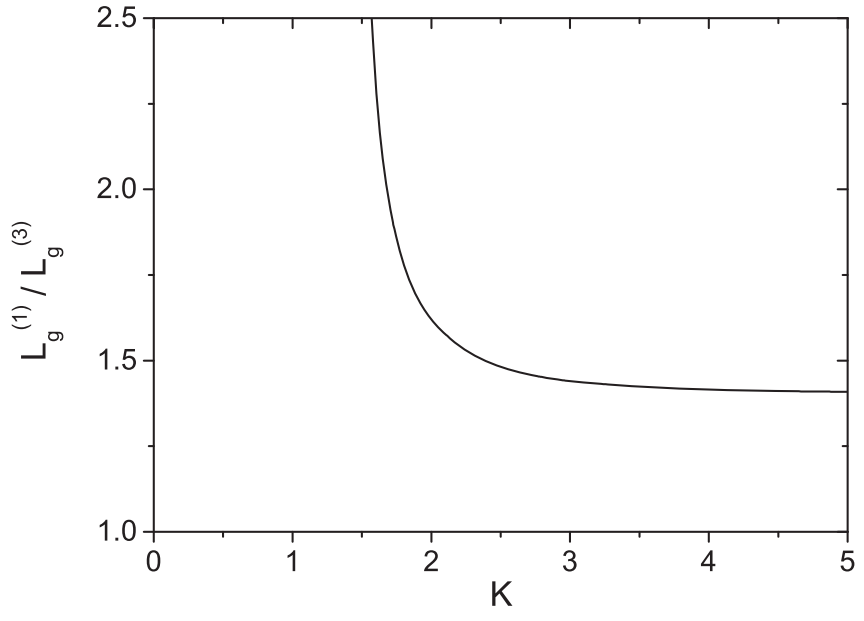

FIG. 2. Ratio of the gain length of the retuned fundamental to the gain length of the third harmonic (3) for lasing at the same wavelength versus rms undulator parameter $K$. The ratio is derived in the frame of the three-dimensional theory for an optimized beta-function and negligible energy spread [9].

remains a reserve in the value of parameter $K$ allowing effective lasing at the third harmonic.

Amplification process of harmonics degrades with the increase of the energy spread in the electron beam more rapidly than that of the fundamental. However, in practical situations there is always the range of parameters for which the harmonic lasing still has an advantage [9].

The most attractive feature of the saturated harmonic lasing is that the spectral brightness (or brilliance) of harmonics is comparable to that of the fundamental [9]. Indeed, a good estimate for the saturation efficiency is $\lambda_{\mathrm{w}} /\left(h L_{\mathrm{sat}, h}\right)$, where $L_{\mathrm{sat}, h}$ is the saturation length of a harmonic ( $h=1$ for the fundamental). At the same time, the relative rms bandwidth has the same scaling. In other words, reduction of power is compensated by the bandwidth reduction, and the spectral power remains the same. If we consider the lasing at the same wavelength on the fundamental and on a harmonic (with the retuned undulator parameter $K$ ), transverse coherence properties are about the same since they are mainly defined by the emittance-towavelength ratio [24,25]. Thus, also the spectral brightness is about the same in both cases.

Although known theoretically for a long time [5-8], harmonic lasing in high-gain FELs was never demonstrated experimentally. Moreover, it was never considered for practical applications in X-ray FELs. The situation was changed after publication of Ref. [9] where it was concluded that the harmonic lasing in X-ray FELs is much more robust than usually thought, and can be effectively used in the existing and future X-ray FELs. In particular, the European XFEL [26] can greatly outperform the specifications in terms of the highest possible photon energy: it can reach $60-100 \mathrm{keV}$ range for the third harmonic lasing. It was also shown [27] that one can keep 
sub-Ångström range of operation of the European XFEL after CW upgrade of the accelerator with a reduction of electron energy from $17.5 \mathrm{GeV}$ to $7 \mathrm{GeV}$. Another application of harmonic lasing is a possible upgrade of FLASH [28] with the aim to increase the photon energy up to $1 \mathrm{keV}$ with the present energy $1.25 \mathrm{GeV}$ of the accelerator. To achieve this goal, one should install a specially designed undulator optimized for the third harmonic lasing as suggested in [29].

\section{HARMONIC LASING SELF-SEEDED FEL}

A poor longitudinal coherence of SASE FELs [30-32] stimulated efforts for its improvement. Since an external seeding seems to be difficult to realize in the X-ray regime, a so-called self-seeding has been proposed [15-17]. There are alternative approaches for reducing bandwidth and increasing spectral brightness of X-ray FELs without using optical elements. One of them [33,34] suggests to use chicanes inside the undulator system to increase slippage of the radiation and to establish long-range correlations in the radiation pulse. Another method was proposed in [9] and is based on the combined lasing on a harmonic in the first part of the undulator [with increased undulator parameter $K$, see formula (2)] and on the fundamental in the second part. In this way the second part of the undulator is seeded by a narrow-band signal generated via a harmonic lasing in the first part. This concept was named HLSS FEL (harmonic lasing self-seeded FEL) 3 . Note that a very similar concept was proposed in [35] and was called a purified SASE FEL, or pSASE.

Typically, gap-tunable undulators are planned to be used in X-ray FEL facilities. If maximal undulator parameter $K$ is sufficiently large, the concept of harmonic lasing selfseeded FEL can be applied in such undulators (see Fig. 3). An undulator is divided into two parts by setting two different undulator parameters such that the first part is tuned to a $h$ th subharmonic of the second part which is tuned to a wavelength of interest $\lambda_{1}$. Harmonic lasing occurs in the exponential gain regime in the first part of the undulator, also the fundamental in the first part stays well below saturation. In the second part of the undulator the fundamental is resonant to the wavelength, previously amplified as the harmonic. The amplification process proceeds in the fundamental up to saturation. In this case the bandwidth is defined by the harmonic lasing (i.e. it is

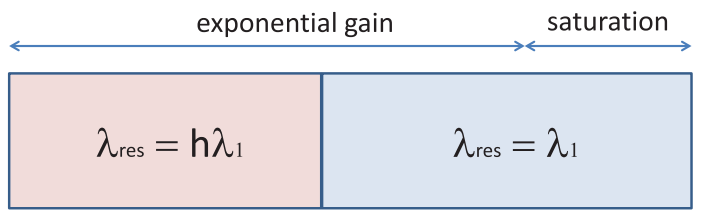

FIG. 3. Conceptual scheme of a harmonic lasing self-seeded FEL. reduced by a significant factor depending on harmonic number) but the saturation power is still as high as in the reference case of lasing at the fundamental in the whole undulator, i.e. the spectral brightness increases.

The enhancement factor of the coherence length (or, bandwidth reduction factor), that one obtains in HLSS FEL in comparison with a reference case of lasing in SASE FEL mode in the whole undulator, reads 3 :

$$
R \simeq h \frac{\sqrt{L_{\mathrm{w}}^{(1)} L_{\mathrm{sat}, h}}}{L_{\mathrm{sat}, 1}}
$$

Here $h$ is harmonic number, $L_{\text {sat }, 1}$ is the saturation length in the reference case of the fundamental lasing with the lower $K$-value, $L_{\mathrm{w}}^{(1)}$ is the length of the first part of the undulator, and $L_{\mathrm{sat}, h}$ is the saturation length of harmonic lasing. We notice that it is beneficial to increase the length of the first part of the undulator. Since it must be shorter than the saturation length of the fundamental harmonic in the first section, one can consider delaying the saturation of the fundamental with the help of phase shifters $[8,9]$ in order to increase $L_{\mathrm{w}}^{(1)}$. However, for the sake of simplicity, we did not use this option in our experiments.

Despite the bandwidth reduction factor (4) being significantly smaller than that of self-seeding schemes using optical elements [15-17], the HLSS FEL scheme is very simple and robust, and it does not require any additional installations, i.e. it can always be used in existing or planned gap-tunable undulators with a sufficiently large $K$-value.

One more advantage of the HLSS FEL scheme over the SASE FEL (and in many cases over a self-seeded FEL) is the possibility of a more efficient use of a post-saturation taper [36-38] for an improved conversion of the electron beam power to the FEL radiation power [14,39]. It is wellknown [40] that a seeded (self-seeded) FEL works better in the post-saturation tapering regime than SASE FEL. In the latter case, a poor longitudinal coherence limits the length of the tapered part of the undulator to a length on which a slippage of the radiation with respect to the electron beam is comparable with the FEL coherence length $[41,42]$. In a self-seeded FEL the coherence length is much larger and it does not limit the performance of the tapered FEL (unless a sideband instability starts playing a role [36]). A disadvantage of a self-seeded FEL is that the saturation length is almost doubled with respect to the SASE regime, so that the available length for tapering the undulator may become too short. Considering now the HLSS FEL, we can state that it combines both advantages: coherence length is significantly larger than in the case of the SASE FEL, and the saturation length is shorter than that of the SASE FEL. In other words, there is more undulator length, available for tapering, than in the cases of the self-seeded FEL and SASE, and the longitudinal coherence is good enough to 
perform efficient tapering over this length. This makes us believe that HLSS FEL will become a standard mode of operation of X-ray FEL facilities.

Numerical simulations of the HLSS FEL were presented in 3 for the European XFEL [26] and in [39] for FLASH [28]. In this paper we report on the first operation of the harmonic lasing self-seeded FEL. The experiment was performed at the 2nd undulator line of the free electron laser FLASH $[1,23,28]$. We detected clear evidence of the $3 \mathrm{rd}$ harmonic lasing in the wavelength range from $4.5 \mathrm{~nm}$ to $15 \mathrm{~nm}$ and compared performance of HLSS FEL and SASE FEL. Obtained experimental results are in good agreement with expectations [14,39]: HLSS FEL provides more powerful photon beams with improved longitudinal coherence.

\section{OPERATION OF THE HLSS FEL AT FLASH2}

The first soft X-ray FEL user facility FLASH $[1,28]$ was upgraded to split the electron pulse trains between the two undulator lines so that the accelerator with maximum energy of $1.25 \mathrm{GeV}$ now drives both lines. In a new separate tunnel, a second undulator line, called FLASH2, with a variable-gap undulator was installed, while a new experimental hall has space for up to six experimental stations [23]. The gap-tunable undulator of FLASH2 consists of twelve $2.5 \mathrm{~m}$ long sections with the undulator period of $3.14 \mathrm{~cm}$ and the maximum rms $K$-value about 1.9 . This makes it possible [see formula (2)] to study the HLSS FEL scheme with the 3rd harmonic seeding. Due to the parallel operation with FLASH1 undulator line, the invasive electron beam diagnostics, placed in the common part of the machine, was not available during the measurements. Moreover, FLASH2 is not equipped with the longitudinal beam diagnostics yet. For this reasons we cannot directly compare our measurements with numerical simulations. We could, however, observe a good qualitative agreement with the simulations [39] that were done before the measurements.

\section{A. First lasing at $7 \mathbf{~ n m}$}

On May 1, 2016 we were able to successfully perform the first test of HLSS FEL at FLASH2. Electron energy was $948 \mathrm{MeV}$, charge $0.4 \mathrm{nC}$. Initially we tuned 10 undulator sections to a standard SASE, operating in the exponential gain regime at the wavelength of $7 \mathrm{~nm}$ ( $\mathrm{rms} K$ parameter was 0.73 ); the pulse energy was $12 \mu \mathrm{J}$. Then we detuned the first section, tuned it to the third subharmonic (rms K was 1.9) and scanned it around $21 \mathrm{~nm}$. We repeated the measurements with the first two sections, and then with the first three sections. Note that the fundamental at $21 \mathrm{~nm}$ was also in the exponential gain regime, pulse energy after three undulator sections was $40 \mathrm{~nJ}$, i.e. it was far away from saturation (which was achieved at the $200 \mu \mathrm{J}$ level). This

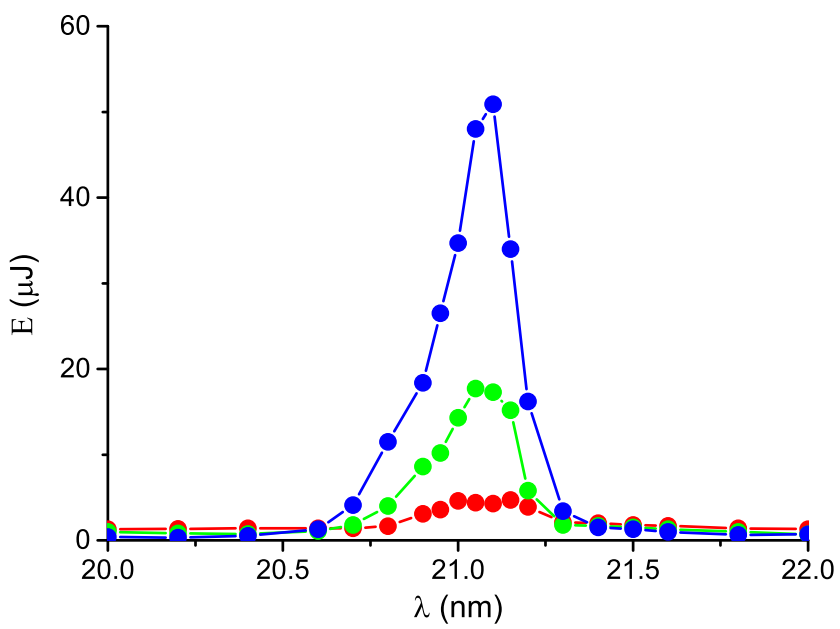

FIG. 4. Scan of the resonance wavelength of the first part of the undulator consisting of one undulator section (red), two sections (green), and three sections (blue). Pulse energy is measured after the second part of the undulator tuned to $7 \mathrm{~nm}$.

means, in particular, that the nonlinear harmonic generation in the first part of the undulator is excluded.

One can see from Fig. 4 that the effect is essentially resonant. For example, in the case when three undulator sections were scanned, the ratio of pulse energies at the optimal tune, $21.1 \mathrm{~nm}$, and at the tune of $20 \mathrm{~nm}$ is $51 \mu \mathrm{J} / 0.3 \mu \mathrm{J}=170$. This ratio is likely underestimated because the background radiation at the fundamental at $20 \mathrm{~nm}$ (even being much weaker, about $40 \mathrm{~nJ}$ ) is more efficiently detected by the microchannel plate (MCP) based detector $[43,44]$ used in this measurement. Note that the $\mathrm{MCP}$ detector has a very large dynamical range and a high signal-to-noise ratio. For these reasons it is best suited to measurements of the FEL gain curve and statistical properties of the FEL radiation $[1,45,46]$. This detector has no absolute calibration, therefore in our experiments we used gas monitor detector (GMD) $[47,48]$ to absolutely calibrate the MCP detector at the level of $10 \mu \mathrm{J}$.

We claim that there can be only one explanation of the effect that we observe in Fig. 4: FEL gain at $7 \mathrm{~nm}$ is strongly reduced as soon as the first part of the undulator is detuned, and then the gain is recovered (and becomes even larger) due to the 3rd harmonic lasing in the first part as soon as the resonant wavelength is $21 \mathrm{~nm}$.

We should stress that the pulse energy with three retuned undulator sections $(51 \mu \mathrm{J})$ is significantly larger than that in the homogeneous undulator tuned to $7 \mathrm{~nm}$ (it was $12 \mu \mathrm{J}$ ). This is because the gain length of harmonic lasing is shorter than that of the fundamental tuned to the same wavelength (see formula (3), Fig. 2 and Refs. [8,9,14,39]). A rough estimate gives us the ratio of gain lengths about 1.4 which is somewhat smaller than the ratio in Fig. 2. The difference can be explained by a contribution of the energy spread (not included in Fig. 2) and by the fact that the undulator betafunction was larger than an optimum value. 


\section{B. Improvement of the longitudinal coherence at $11 \mathrm{~nm}$}

We continued the studies of the HLSS FEL at FLASH2 in June 2016. Since the electron energy was different $(757 \mathrm{MeV})$, we lased at another wavelength, $11 \mathrm{~nm}$. We also used a different charge, $0.25 \mathrm{nC}$, in this experiment. The undulator settings were similar to the previous case: we used ten undulator modules, rms $K$-parameter was 0.73 in SASE mode and 1.9 in the first part of the undulator in HLSS mode. The difference with the previous measurements was that we detuned four undulator modules in HLSS regime.

In the same way as in the previous experiment, we performed the scan of the $K$ parameter in the first part of the undulator and saw a resonance behavior again. In combination with the fact that the fundamental at $33 \mathrm{~nm}$ was by three orders of magnitude below saturation this proves that we had harmonic lasing at $11 \mathrm{~nm}$ in the first part of the undulator. The pulse energies were $11 \mu \mathrm{J}$ in SASE mode and $53 \mu \mathrm{J}$ in HLSS mode.

The main goal of this run was to demonstrate that HLSS scheme indeed helps to improve the longitudinal coherence of FEL pulses with respect to the standard SASE regime. One can do this by the demonstration of the bandwidth reduction and by the measurements of an increase of the coherence time.

The spectra were measured with the wide-spectral-range XUV spectrometer [49] of FLASH2. A narrow entrance slit is imaged by a $12001 / \mathrm{mm}$ spherical variable line spacing grating in the 5th grating order which allows for a resolution better than $0.01 \mathrm{~nm}$. In Fig. 5 we present the averaged spectra for two study cases: SASE FEL with ten undulator modules and HLSS FEL with four modules tuned to $33 \mathrm{~nm}$ and six modules tuned to $11 \mathrm{~nm}$. Let us note that a per cent level discrepancy between the measured wavelength (about $10.9 \mathrm{~nm}$ ) and the wavelength expected by the undulator server $(11 \mathrm{~nm})$ comes from the fact that the server

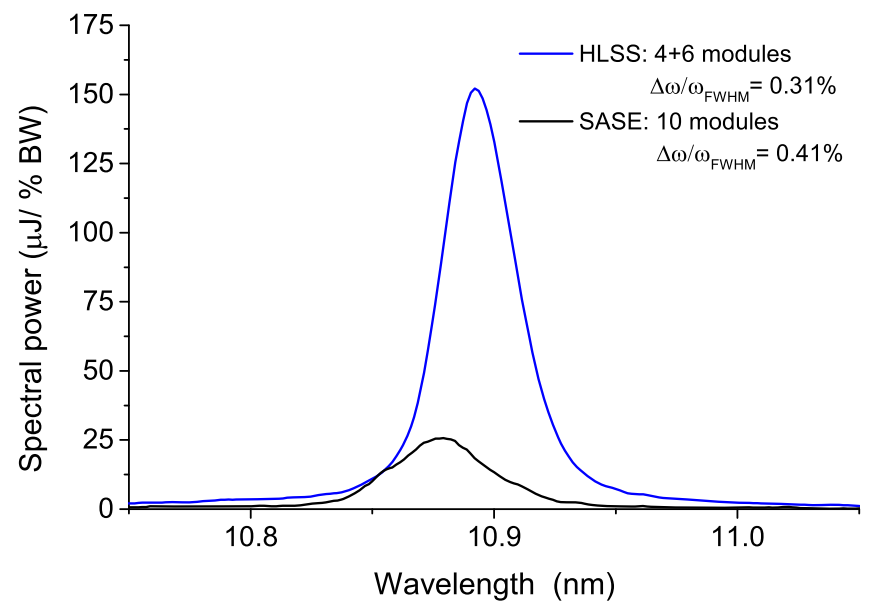

FIG. 5. Spectral density of the radiation energy for HLSS FEL configuration (blue) and for SASE FEL (black). uses electron energy calculated from the rf vector sum and not from a direct measurement of the electron beam energy. However, the redshift of the radiation for the HLSS configuration with respect to the SASE case is real and can be explained by the fact that a seeded FEL radiates more efficiently in the case of a small redshift [32].

The spectra in Fig. 5 are the results of averaging over 50 single-shot spectra in each case. One can see that HLSS FEL indeed has a smaller bandwidth, $0.31 \%$, as compared to $0.41 \%$ in the case of SASE FEL. The bandwidth reduction factor is 1.3 from this measurement. The spectral power, however, differs by a factor of six due to an additional increase of pulse energy in HLSS regime. This happens because the 3rd harmonic lasing at $11 \mathrm{~nm}$ has a shorter gain length than lasing at the same wavelength on the fundamental.

An expected bandwidth reduction factor (or coherence enhancement factor) $R$ from formula (4) can be estimated at 1.7. The discrepancy can in a general case be explained by the energy jitter and/or energy chirp in the electron beam. The energy jitter effect is supposed to give a small contribution to the spectrum broadening since the FLASH accelerator was quite stable during the measurement, the energy stability can be estimated at the level of a few $10^{-4}$. A contribution of the energy chirp, however, being converted to a frequency chirp within an FEL pulse, can be significant. The energy chirp appears in the accelerator on the one hand due to off-crest acceleration, needed for the bunch compression in magnetic chicanes, and on the other hand due to collective self-fields in the bunch (wakefields, longitudinal space charge) [1]. Both contributions can partially or fully compensate each other, this depends on accelerator settings. In the experiment we could tweak the bunch compression, trying to minimize the HLSS FEL bandwidth, and we succeeded partially, the energy chirp was reduced to the $0.1 \%$ level.

Another method of determination of an improvement of the longitudinal coherence (independent of the presence of the frequency chirp in FEL pulses) is based on statistical measurements of the FEL pulse energy along the undulator length. It is well known [31,32] that in high-gain linear regime the radiation from a SASE FEL has a statistics of a completely chaotic polarized light [50]. Shot-to-shot rms fluctuations of the FEL pulse energy $\sigma$ are connected with the number of modes by a simple relation: $M=1 / \sigma^{2}$. Number of modes can be represented as a product of the numbers of longitudinal, $M_{L}$, and transverse, $M_{T}$, modes. The latter is usually close to one, $M_{T} \simeq 1.1-1.2$ when a SASE FEL is well designed and optimized $[24,25]$. If one uses a small aperture to select only the central part of the FEL beam, the pulse energy fluctuations are a measure of the number of the longitudinal modes [45]: $M_{L}=1 / \sigma^{2}$. For a given FEL pulse length, the coherence length $L_{\mathrm{coh}}$ is inversely proportional to the number of the longitudinal modes, $M_{L}$. Making a reasonable assumption that the FEL 
pulse length is the same in both cases, HLSS and SASE, we end up with a simple ratio of coherence lengths for these two cases:

$$
R=\frac{L_{\mathrm{coh}}^{\mathrm{HLSS}}}{L_{\mathrm{coh}}^{\mathrm{SASE}}} \simeq \frac{M_{L}^{\mathrm{SASE}}}{M_{L}^{\mathrm{HLSS}}}=\frac{\sigma_{\mathrm{HLSS}}^{2}}{\sigma_{\mathrm{SASE}}^{2}}
$$

In Fig. 6 we present the measurements of the FEL pulse energy and its fluctuations versus undulator length for a small aperture (significantly smaller than the FEL beam size) in front of the MCP detector. The measurements start behind the sixth undulator section because at this position the contribution of the background radiation at $33 \mathrm{~nm}$ is already negligible. In both cases, HLSS and SASE, the maximum of pulse energy fluctuations is achieved within the part of the undulator where the measurements were performed. However, in HLSS case the fluctuations drop down more significantly because the FEL enters nonlinear
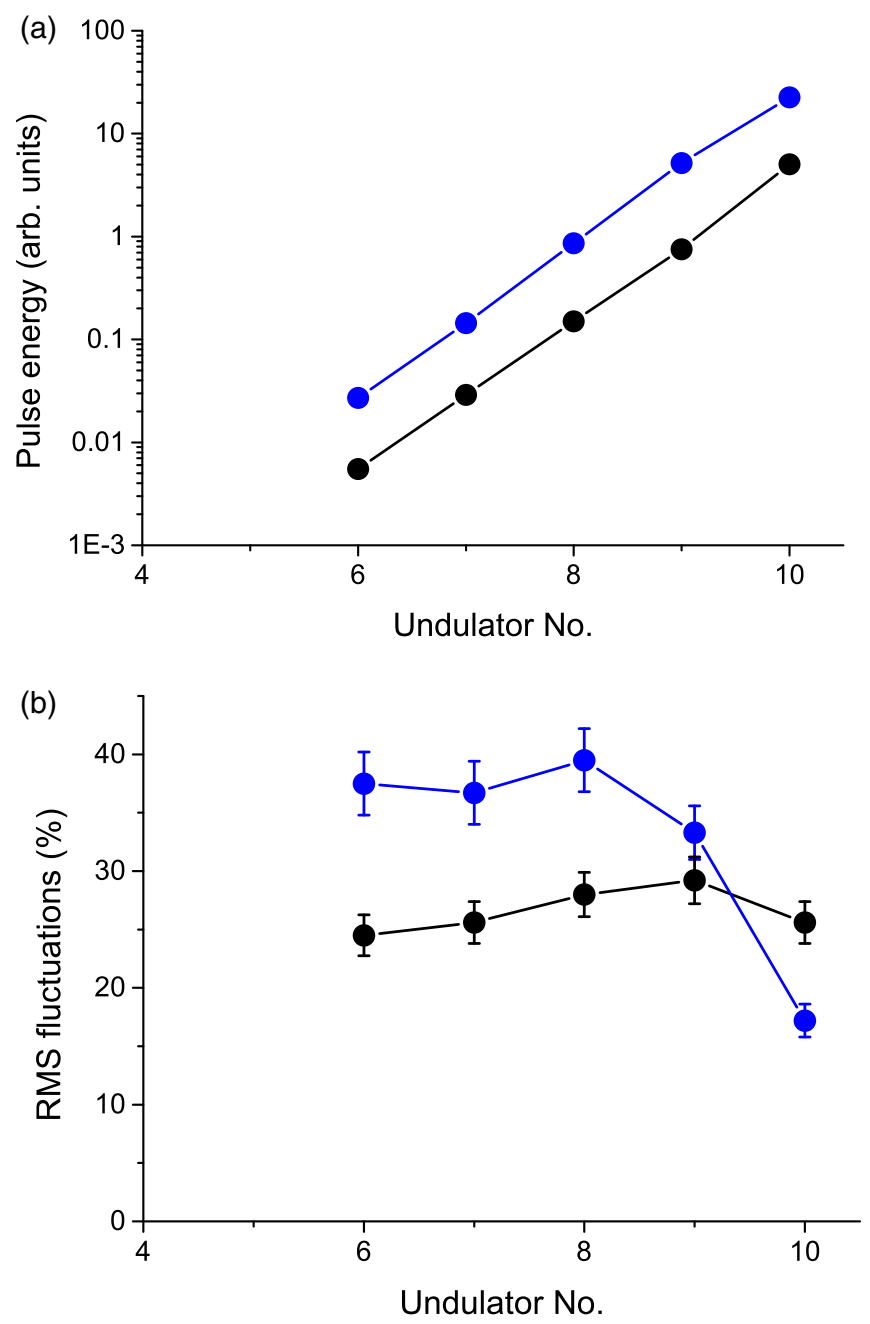

FIG. 6. Radiation pulse energy (left plot) and pulse energy fluctuations (right plot) in the second part of the undulator for HLSS (blue) and for SASE (black). Small aperture in front of the MCP detector is used in this measurement. stage of amplification in this case. As one can see, in the linear regime of the FEL operation (Secs. 6 to 8) the fluctuations for HLSS case are visibly larger than in the SASE case. The validity of an assumption that pulse length in both cases is the same is justified by the fact that both FEL configurations were driven by the same electron beam under the same conditions. We did the measurements twice for each configuration to make sure that the results are not affected by any drifts in the accelerator. Thus, we can conclude that in the HLSS case we had a smaller number of modes, or a larger coherence length. Using formula (5) with the fluctuations measured behind the 8th undulator section for HLSS and the 9th section for SASE (at these positions with the largest fluctuations we have a similar gain in both modes), we obtain an estimate for the coherence enhancement factor in the end of the exponential gain regime: $R \simeq 1.8 \pm 0.3$. This is in a good agreement with already presented theoretical estimate $R \simeq 1.7$ obtained from (4).

Note that this moderate enhancement, observed in our experiment, is obtained because we are limited to application of the third (and not higher) harmonic at FLASH2. Further improvement can be done by increasing the length of the first part of the undulator [see formula (4)], making sure that the fundamental in the first part stays well below saturation (one can delay the saturation by using phase shifters as suggested in $[8,9])$. In a gap-tunable undulator with a higher $K$, like SASE3 undulator of the European XFEL (with the rms $K$ about 7), one can, in principle, use a much higher harmonic number thus expecting a much higher coherence enhancement factor.

\section{A more efficient post-saturation taper at $15 \mathbf{~ n m}$}

In November 2016 we set up HLSS FEL as a configuration with four first undulators tuned to $45 \mathrm{~nm}$ and the last eight undulators tuned to $15 \mathrm{~nm}$. The electron energy was $645 \mathrm{MeV}$, the charge was $100 \mathrm{pC}$, the rms value of $K$ was 1.9 in the first part of the undulator and 0.73 in the second part. We reached FEL saturation in SASE and HLSS modes, and applied post-saturation taper to improve FEL efficiency [36-38].

Post-saturation taper in FLASH2 undulator is implemented as a step-taper (i.e. the undulator $K$ changes from section to section but is constant within a section) with linear or quadratic law. We used quadratic taper and for each mode (HLSS and SASE) optimized two parameters: beginning of tapering and the taper depth. We ended up with the following optimized parameters: beginning of tapering was in the 9th (10th) undulator and the taper depth was $0.9 \%(0.7 \%)$ for HLSS (SASE). Pulse energy was enhanced for HLSS configuration from $18 \mu \mathrm{J}$ in nontapered undulator to $31 \mu \mathrm{J}$ when post-saturation taper was applied. In case of SASE FEL the respective enhancement was from $15 \mu \mathrm{J}$ to $20 \mu \mathrm{J}$. The pulse energy versus undulator length for both operation modes is presented in Fig. 7. 


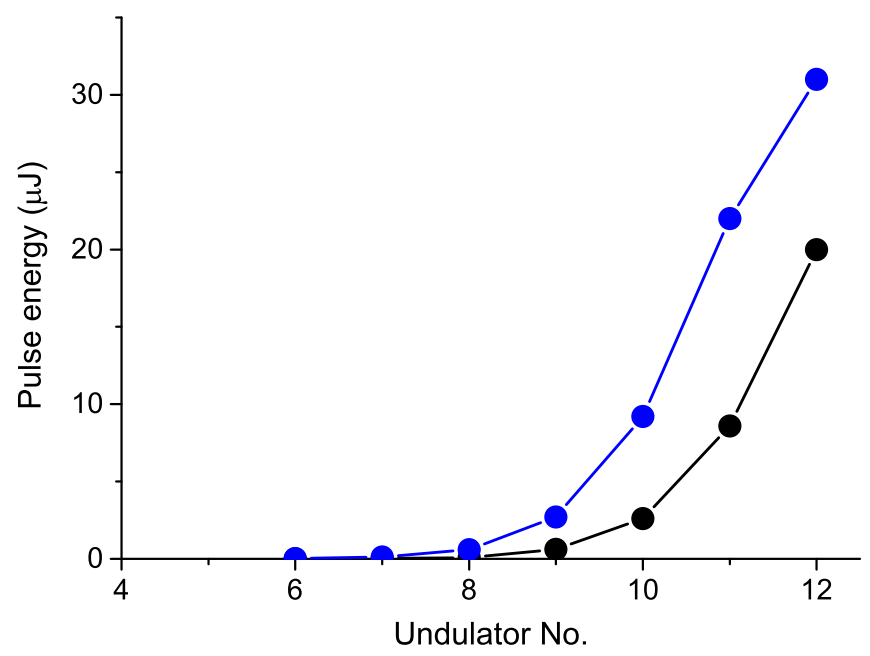

FIG. 7. Radiation pulse energy versus position in the undulator for HLSS (blue) and for SASE (black). Post-saturation taper was optimized for both cases.

Note that a similar efficiency enhancement was previously observed in numerical simulations [14,39]. As it was discussed above, the improvement of post-saturation taper regime is achieved in HLSS case for two reasons: an earlier saturation and a better longitudinal coherence than in the SASE case.

\section{Lasing at $4.5 \mathrm{~nm}$}

In September 2016 we were able to drive HLSS FEL by the electron beam with a higher energy, $1080 \mathrm{MeV}$, and thus obtain lasing at $4.5 \mathrm{~nm}$ in HLSS configuration. Initially, we tuned the SASE regime with 12 active undulator sections (rms $K$ value was 0.53 ), and could establish an onset of saturation with pulse energy at the level of $20 \mu \mathrm{J}$. Then we tuned the first three sections to

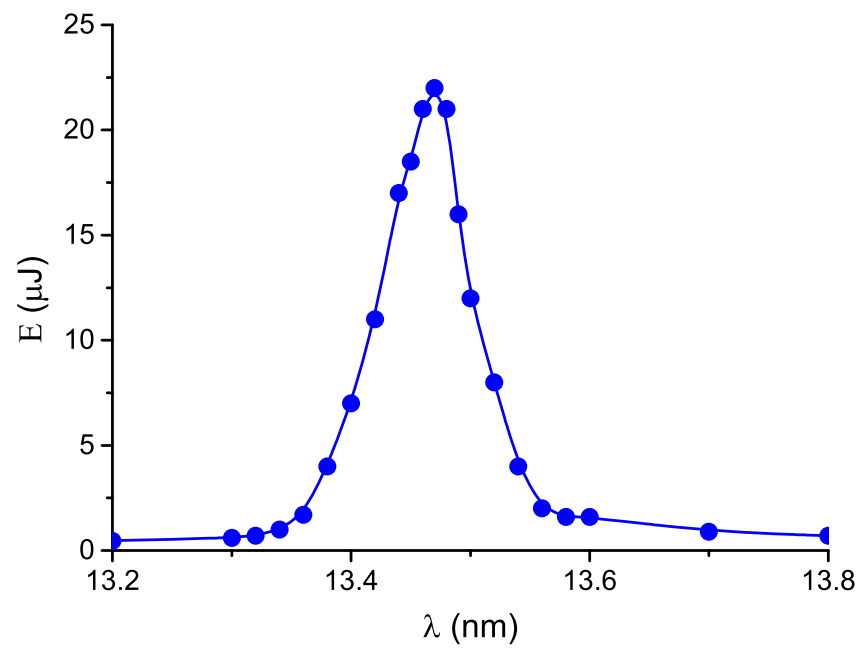

FIG. 8. Scan of the resonance wavelength of the first part of the undulator consisting of three undulator sections. Pulse energy is measured after the second part of the undulator tuned to $4.5 \mathrm{~nm}$ and operated close to the FEL saturation.
$13.5 \mathrm{~nm}$ (increasing rms $K$ value to 1.69), thus providing the third harmonic signal at $4.5 \mathrm{~nm}$ for seeding the last nine undulators. The scan of the undulator tune of the first three modules is presented in Fig. 8. The resonant behavior together with the fact that the fundamental at $13.5 \mathrm{~nm}$ was more than three orders of magnitude below saturation proves that we had the third harmonic lasing at $4.5 \mathrm{~nm}$ in the first part of the undulator.

\section{CONCLUSION}

We were able to successfully demonstrate the harmonic lasing phenomena and the HLSS FEL principle at FLASH2 in the wavelength range between 4.5 and $15 \mathrm{~nm}$. A change from SASE to HLSS configuration was very simple and fast, it worked well independently of a wavelength and accelerator settings. We can, therefore, forecast that HLSS may become a standard mode of operation of the X-ray FEL user facilities with gap-tunable undulators, providing an improvement of the longitudinal coherence, a reduction of the saturation length and a possibility of a more efficient post-saturation tapering.

It is also important to note that the first evidence of harmonic lasing in a high-gain FEL and at a short wavelength (down to $4.5 \mathrm{~nm}$ ) paves the way for a variety of applications of this effect in X-ray FEL facilities $[9,14,27,29]$.

\section{ACKNOWLEDGMENTS}

We are grateful to the FLASH team for technical support. We would like to thank R. Brinkmann, J. Schneider, E. Weckert, and W. Wurth for valuable discussions and support of this work.

[1] W. Ackermann et al., Operation of a free-electron laser from the extreme ultraviolet to the water window, Nat. Photonics 1, 336 (2007).

[2] P. Emma et al., First lasing and operation of an ångstromwavelength free-electron laser, Nat. Photonics 4, 641 (2010).

[3] T. Ishikawa et al., A compact X-ray free-electron laser emitting in the sub-ångström region, Nat. Photonics 6, 540 (2012).

[4] A. M. Kondratenko and E. L. Saldin, Generating of coherent radiation by a relativistic electron beam in an ondulator, Part. Accelerators 10, 207 (1980).

[5] J. B. Murphy, C. Pellegrini, and R. Bonifacio, Collective instability of a free electron laser including space charge and harmonics, Opt. Commun. 53, 197 (1985).

[6] R. Bonifacio, L. De Salvo, and P. Pierini, Large harmonic bunching in a high-gain free-electron laser, Nucl. Instrum. Methods Phys. Res., Sect. A 293, 627 (1990).

[7] Z. Huang and K. Kim, Three-dimensional analysis of harmonic generation in high-gain free-electron lasers, Phys. Rev. E 62, 7295 (2000). 
[8] B. W. J. McNeil, G. R. M. Robb, M. W. Poole, and N. R. Thompson, Harmonic Lasing in a Free-Electron-Laser Amplifier, Phys. Rev. Lett. 96, 084801 (2006).

[9] E. A. Schneidmiller and M. V. Yurkov, Harmonic lasing in x-ray free electron lasers, Phys. Rev. ST Accel. Beams 15, 080702 (2012).

[10] H. Freund, S. Biedron, and S. Milton, Nonlinear harmonic generation and proposed experimental verification in SASE FELs, Nucl. Instrum. Methods Phys. Res., Sect. A 445, 53 (2000).

[11] E. L. Saldin, E. A. Schneidmiller, and M. V. Yurkov, Properties of the third harmonic of the radiation from self-amplified spontaneous emission free electron laser, Phys. Rev. ST Accel. Beams 9, 030702 (2006).

[12] A. Tremaine, X. J. Wang, M. Babzien, I. Ben-Zvi, M. Cornacchia, H.-D. Nuhn, R. Malone, A. Murokh, C. Pellegrini, S. Reiche, J. Rosenzweig, and V. Yakimenko, Experimental Characterization of Nonlinear Harmonic Radiation from a Visible Self-Amplified Spontaneous Emission Free-Electron Laser at Saturation, Phys. Rev. Lett. 88, 204801 (2002).

[13] D. Ratner et al., Second and third harmonic measurements at the linac coherent light source, Phys. Rev. ST Accel. Beams 14, 060701 (2011).

[14] E. A. Schneidmiller and M. V. Yurkov, Harmonic lasing self-seeded FEL, in Proceedings of FEL2013, New York, USA, 2013 (JACoW, New York, 2013), p. 700, http://www .jacow.org.

[15] J. Feldhaus, E. L. Saldin, J. R. Schneider, E. A. Schneidmiller, and M. V. Yurkov, Possible application of X-ray optical elements for reducing the spectral bandwidth of an X-ray SASE FEL, Opt. Commun. 140, 341 (1997).

[16] E. L. Saldin, Y. V. Shvyd'ko, E. A. Schneidmiller, and M. V. Yurkov, X-ray FEL with a meV bandwidth, Nucl. Instrum. Methods Phys. Res., Sect. A 475, 357 (2001).

[17] G. Geloni, V. Kocharyan, and E. L. Saldin, A novel selfseeding scheme for hard X-ray FELs, J. Mod. Opt. 58, 1391 (2011).

[18] W. B. Colson, The nonlinear wave equation for higher harmonics in free-electron lasers, IEEE J. Quantum Electron. 17, 1417 (1981).

[19] S. V. Benson and J. M. J. Madey, Demonstration of harmonic lasing in a free-electron laser, Phys. Rev. A 39, 1579 (1989).

[20] R. W. Warren, L. C. Haynes, D. W. Feldman, W. E. Stein, and S. J. Gitomer, Lasing on the third harmonic, Nucl. Instrum. Methods Phys. Res., Sect. A 296, 84 (1990).

[21] R. Hajima, R. Nagai, N. Nishimori, N. Kikuzawa, and E. J. Minehara, Third-harmonic lasing at JAERI-FEL, Nucl. Instrum. Methods Phys. Res., Sect. A 475, 43 (2001).

[22] N. Sei, H. Ogawa, and K. Yamada, Third harmonic lasing in a storage ring free-electron laser, J. Phys. Soc. Jpn. 79, 093501 (2010).

[23] B. Faatz et al., Simultaneous operation of two soft x-ray free-electron lasers driven by one linear accelerator, New J. Phys. 18, 062002 (2016).

[24] E. L. Saldin, E. A. Schneidmiller, and M. V. Yurkov, Coherence properties of the radiation from X-ray free electron laser, Opt. Commun. 281, 1179 (2008).
[25] E. L. Saldin, E. A. Schneidmiller, and M. V. Yurkov, Statistical and coherence properties of radiation from X-ray free-electron lasers, New J. Phys. 12, 035010 (2010).

[26] M. Altarelli et al., The European X-ray free-electron laser, DESY Technical Design Report, Preprint No. DESY 2006097, 2006 (see also http://xfel.desy.de).

[27] R. Brinkmann, E. A. Schneidmiller, J. Sekutowicz, and M. V. Yurkov, Prospects for CW and LP operation of the European XFEL in hard X-ray regime, Nucl. Instrum. Methods Phys. Res., Sect. A 768, 20 (2014).

[28] S. Schreiber and B. Faatz, The free-electron laser FLASH, High Power Laser Sci. Eng. 3, e20 (2015).

[29] E. A. Schneidmiller and M. V. Yurkov, A possible upgrade of FLASH for harmonic lasing down to $1.3 \mathrm{~nm}$, Nucl. Instrum. Methods Phys. Res., Sect. A 717, 37 (2013).

[30] R. Bonifacio, C. Pellegrini, and L. M. Narducci, Collective instabilities and high-gain regime in a free electron laser, Opt. Commun. 50, 373 (1984).

[31] E. L. Saldin, E. A. Schneidmiller, and M. V. Yurkov, Statistical properties of radiation from VUV and X-ray free electron laser, Opt. Commun. 148, 383 (1998).

[32] E. L. Saldin, E. A. Schneidmiller, and M. V. Yurkov, The Physics of Free Electron Lasers (Springer, Berlin, 1999).

[33] J. Wu, J. A. Marinelli, and C. Pellegrini, Generation of longitudinally coherent ultra high power X-ray FEL pulses by phase and amplitude mixing, in Proceedings of the 32th International Free Electron Laser Conference, Nara, Japan, 2012 (JACoW, Nara, 2012), p. 23, http://www .jacow.org.

[34] B. W. J. McNeil, N. R. Thompson, and D. J. Dunning, Transform-Limited X-Ray Pulse Generation from a High-Brightness Self-Amplified Spontaneous-Emission Free-Electron Laser, Phys. Rev. Lett. 110, 134802 (2013).

[35] D. Xiang, Y. Ding, Z. Huang, and H. Deng, Purified selfamplified spontaneous emission free-electron lasers with slippage-boosted filtering, Phys. Rev. ST Accel. Beams 16, 010703 (2013).

[36] N. M. Kroll, P. L. Morton, and M. N. Rosenbluth, Freeelectron lasers with variable parameter wigglers, IEEE J. Quantum Electron. 17, 1436 (1981).

[37] W. M. Fawley, "Optical guiding" limits on extraction efficiencies of single-pass, tapered wiggler amplifiers, Nucl. Instrum. Methods Phys. Res., Sect. A 375, 550 (1996).

[38] E. A. Schneidmiller and M. V. Yurkov, Optimization of a high efficiency free electron laser amplifier, Phys. Rev. ST Accel. Beams 18, 030705 (2015).

[39] E. A. Schneidmiller and M. V. Yurkov, Studies of harmonic lasing self-seeded FEL at FLASH2, in Proceedings of IPAC2016, Busan, Korea, 2016 (JACoW, Busan, 2016), p. 725, http://www.jacow.org.

[40] W. M. Fawley, Z. Huang, K.-J. Kim, and N. A. Vinokurov, Tapered undulators for SASE FELs, Nucl. Instrum. Methods Phys. Res., Sect. A 483, 537 (2002).

[41] E. A. Schneidmiller and M. V. Yurkov, The universal method for optimization of undulator tapering in FEL amplifiers, in Proceedings of SPIE 9512, Advances in X-ray Free-Electron Lasers Instrumentation III, 2015 (SPIE, Prague, 2015), p. 951219; . 
[42] E. A. Schneidmiller and M. V. Yurkov, Optimization of a high efficiency free electron laser amplifier, in Proceedings of FEL2015 Conference, Daejeon, Korea, 2015 (JACoW, Daejeon, 2015), p. MOC02, http://www.jacow.org.

[43] A. Bytchkov, A. A. Fateev, J. Feldhaus, U. Hahn, M. Hesse, U. Jastrow, V. Kocharyan, N. I. Lebedev, E. A. Matyushevskiy, E. L. Saldin, E. A. Schneidmiller, A. V. Shabunov, K. P. Sytchev, K. Tiedtke, R. Treusch, and M. V. Yurkov, Development of MCP-based photon diagnostics at the TESLA test facility at DESY, Nucl. Instrum. Methods Phys. Res., Sect. A 528, 254 (2004).

[44] O. Brovko et al., Measurements of ultrasmall charges with MCP detector in flash accelerator, in Proceedings of IPAC2016, Busan, Korea, 2016 (JACoW, Busan, 2016), p. mopow014, http://www.jacow.org.

[45] E. A. Schneidmiller and M. V. Yurkov, Application of statistical methods for measurements of the coherence properties of the radiation from SASE FEL, in Proceedings of IPAC2016, Busan, Korea, 2016 (JACoW, Busan, 2016), p. 738 , http://www.jacow.org.
[46] C. Behrens, N. Gerasimova, C. Gerth, B. Schmidt, E. A. Schneidmiller, S. Serkez, S. Wesch, and M. V. Yurkov, Constraints on photon pulse duration from longitudinal electron beam diagnostics at a soft x-ray free-electron laser, Phys. Rev. ST Accel. Beams 15, 030707 (2012).

[47] K. Tiedtke et al., Gas detectors for x-ray lasers, J. Appl. Phys. 103, 094511 (2008).

[48] K. Tiedtke et al., The soft X-ray free-electron laser FLASH at DESY: beamlines, diagnostics and end-stations, New J. Phys. 11, 023029 (2009).

[49] T. Tanikawa, A. Hage, M. Kuhlmann, J. Gonschior, S. Grunewald, E. Plonjes, S. Dusterer, G. Brenner, S. Dziarzhytski, M. Braune, M. Brachmanski, Z. Yin, F. Siewert, T. Dzelzainis, B. Dromey, M. J. Prandolini, F. Tavella, M. Zepf, and B. Faatz, First observation of SASE radiation using the compact wide-spectral-range XUV spectrometer at FLASH2, Nucl. Instrum. Methods Phys. Res., Sect. A 830, 170 (2016).

[50] J. Goodman, Statistical Optics (John Wiley and Sons, New York, 1985). 